\title{
LA GLOBALIZACION Y LA ARMONIZACIÓN CONTABLE
}

Mg. SABINO TALLA RAMOS

\section{INTRODUCCIÓN}

Hace siglos, cuando el principal transporte era el asno o el caballo, el comercio no podía llegar más allá de unos cuantos kilómetros a la redonda; ahora que un avión recorre en menos de un día los puntos más distantes del planeta, también las mercancías lo pueden hacer. El fax, la comunicación vía satélite, el correo electrónico, internet y la transferencia de fondos en unos segundos de un punto de la tierra a otro, son instrumentos que han propiciado la globalización de la economía. Bajo el término de Globalización entendemos el intercambio generalizado de bienes y servicios entre países y continentes.

La globalización implica un cambio, así los profundos avances que se producen día a día serán compartidos y aceptados por los países, gracias a la difusión e información que se tenga de ellos. La globalización, o internacionalización de todas las actividades humanas, desplazan el concepto de nacionalidad que representó y representa todavía, dependiendo de las etapas en que se encuentren las comunidades del mundo, una etapa más de la evolución del hombre. Antes se formaba un contador con sentido de patria, en los momentos actuales, con sentido de humanidad (Contador Universal).

La globalización y los movimientos de integración; en particular, plantean problemas técnicos de diversa naturaleza. En este trabajo se comenta únicamente la armonización contable.
El creciente ámbito de las operaciones de las empresas, junto con el interés cada vez mayor por este tipo de actividades y por sus consecuencias en la sociedad, han aumentado en los últimos años la demanda de información; y por consiguiente, sus requerimientos a nivel internacional. La mundialización de los mercados y el crecimiento, al mismo nivel de los procesos de inversión, han generado una evolución similar en las necesidades armonizadoras de la información suministrada por las entidades económicas. Para satisfacer esos intereses $y$, en consecuencia, para facilitar la necesaria comparabilidad entre estados financieros emitidos en países con diferentes normativas, se ha comenzado desde hace algunos años un proceso de armonización contable de carácter irreversible.

\section{GLOBALIZACIÓN}

Concepto.- La globalización es un fenómeno que ha adquirido relevancia en los últimos años. Puede describirse como la internacionalización del conocimiento y de las actividades humanas en general. En ese sentido, el fenómeno comprende la internacionalización de las comunicaciones, de la cultura y, fundamentalmente, de la economía y dentro de esta última, del intercambio comercial. Factores políticos y el avance tecnológico, entre otros, han determinado la eliminación práctica de las fronteras físicas y de restricciones formales al movimiento de personas, bienes, imágenes e ideas. En la actualidad, todas las personas y entidades tienen la posibilidad de 
acceder o vincularse "en tiempo real" o con facilidad, a hechos, personas y entidades, ocurridos o localizados en cualquier parte del universo.

Antecedentes.- La globalización, en varios aspectos, no es algo nuevo. Desde los fenicios y hasta la crisis financiera de 1929 , el comercio se desarrolló libre y crecientemente; lo nuevo vino después de la Segunda Guerra Mundial. Diversos eventos políticos acaecidos al final de la década del 80 en Europa, junto con nuevas estrategias empresariales y el tremendo desarrollo tecnológico, han puesto nuevamente en primer plano la importancia del comercio internacional para el desarrollo de los países y han exaltado consecuentemente, la conveniencia de adoptar políticas económicas abiertas con la finalidad de promoverlo. Por otra parte, una nueva concepción sobre los papeles del estado y del sector privado en la economía, han dado impulso a la idea del intercambiocomercial sin fronteras como una de las manifestaciones más notorias de la globalización.

En 1992 se cumplió 500 años de globalización, otrora llamada colonización; antes con carabelas, espadas y cruces, hoy con parabólicas, fuerzas de despliegue rápido y neoliberalismo.

Ventajas y desventajas.- Una de las ventajas indudables de la globalización es, sin duda, la posibilidad de racionalizar los procesos productivos. De este modo, se logra la mejor utilización de los factores y con ello, la reducción de los costos. Estos procedimientos deberían llevar a incentivar la competitividad, al mejoramiento de la calidad y a la rebaja de precios, con beneficios directos para los consumidores.

Entre las desventajas comúnmente atribuidas se mencionan, principalmente, la eliminación de la protección de las industrias domésticas, lo que puede determinar en el corto plazo el cierre de muchas de ellas, imposibilitadas de enfrentar la competencia externa y como resultado, el aumento del desempleo. La orientación en el manejo de la política económica genera un alto costo social, por lo que sería necesario evaluar el rumbo de la política económica para evitar que las ventajas de la globalización se tornen en desventajas.

Efectos de la globalización.- Para nadie pasa desapercibido que los problemas económicos por los que atraviesa el Perú tienen, además de los signos habituales que caracterizan a los países subdesarrollados, un ingrediente más: el fenómeno de la globalización. Si para los países del "primer mundo", la globalización les ha traído ventajas, también les ha sumado problemas, tal como lo demuestra lo acontecido en los países asiáticos y de Europa del Este. Ahora bien, si esto es así, la pregunta es ¿qué se puede esperar de países como el nuestro, de economías primarias y signados por su inestabilidad política y social? Es evidente que en los países emergentes, la vulnerabilidad es mayor frente a este fenómeno y los problemas se multiplican.

Por tal motivo, también no dejan de tener razón quienes aseguran que la crisis actual en el Perú, expresada en la caída de la producción, recesión, el sube y baja de las reservas, el déficit en la balanza comercial y en la cuenta corriente, etc., tengan como base esta nueva realidad: un mundo cada vez más integrado e interdependiente, en donde a decir de un experto-"un estomudo en China puede indicarnos que Argentina está con gripe".

En consecuencia, si bien se trata de un fenómeno exógeno, la globalización, además de sus enormes ventajas, trae también consigo que los problemas ajenos se sientan como propios por la inestabilidad interna que 
generan en estos países, lo que a su vez, volatiliza los planes y proyecciones de crecimiento que puedan hacer los gobiernos y las empresas.

El teórico de la dependencia, el sociólogo y economista Teothonio Do Santos, quien recientemente visitó el Perú, asegura que la globalización no ha roto la cadena de la dependencia, sino que la ha vuelto más sofisticada, porque el capital financiero, que ahora resulta predominante y que no genera necesariamente producción ni desarrollo, se ha vuelto más volátil que nunca. Esta situación impide a países como los nuestros ser capaces de potenciar su industria y menos a desarrollarse, con lo cual la brecha que nos separa de las naciones ricas tiende a profundizarse.

Dentro de este escenario, se debe tener en cuenta el surgimiento y desarrollo de movimientos de integración regional, como un aspecto estrechamente vinculado al proceso de globalización. Si bien los movimientos de integración regional tienen como objetivo inmediato una liberalización del comercio entre sus países miembros, sus proyecciones de futuro apuntan a su internacionalización, con lo cual se han de transformar en un elemento fundamental de la globalización.

La prueba contundente de "no retroceso" del proceso de globalización es la constitución de los bloques económicos regionales como, por ejemplo, la Unión Europea y los Tigres Asiáticos, en Europa y Asia; y el NAFTA (Tratado de libre Comercio), GRAN (el Pacto Andino), el Mercado Común Centroamericano, CARICOM (La Comunidad del Caribe), y el MERCOSUR (Mercado Común del Sur) en el continente americano, entre otros.

Desde el punto de vista profesional, la globalización implica un esfuerzo adicional para capacitarse y conocer los adelantos tecnológicos referentes a nuestra especialidad, no tan sólo de nuestra ciudad, sino de todas partes.

\section{ARMONIZACIÓN CONTABLE}

- Contabilidad en globalización.- La contabilidad en una economía globalizada permitirá el acceso a la información financiera internacional, a otros mercados, al desarrollo industrial, cumpliendo asimismo el rol de suministrar la información para una adecuada toma de decisiones. La contabilidad cumplirá estos objetivos, en la medida que provea de información que pueda ser entendida en forma uniforme y comparativa en los diferentes países y por distintos organismos. Para lograr este objetivo, deberá apoyarse en normas comunes de aceptación universal, que deben ser establecidas en el país con características propias, y que al mismo tiempo, permitan su comparabilidad en el marco de la globalización económica mundial.

Esto plantea hoy un verdadero desafío para la profesión contable: la armonización de las normas y prácticas contables para la elaboración y revisión independiente de estados financieros. La contabilidad es un sistema de información, debe contener las cualidades básicas de este tipo de sistemas para cumplir adecuadamente su función. De éstas, la utilidad constituye una cualidad esencial sobre la que no hay discusión, y para lograr dicha utilidad a nivel supranacional, la homogeneización es un requerimiento ineludible. El lenguaje utilizado en la contabilidad responde a los usos y costumbres de los países, traducido en una armonización de principios contables y terminología común.

- Armonización contable.- De acuerdo al Diccionario de la Lengua Española, armonizar significa "poner en armonía o 
hacer que no discuerden o se rechacen dos o más partes de un todo o dos o más cosas que deben concurrir al mismo fin", la armonización sería "el poner de acuerdo, el conciliar las normas existentes". Es el proceso de conciliación de las diferentes prácticas de los países que pueden alcanzar una uniformidad y que ha conllevado a una normalización contable que no sea rígida.

Al hablar de la armonización de la información financiera, su sociedad o comunidad dejó de ser todo lo que ocurra dentro de las fronteras de su país a la que ésta pertenezca.

\section{- Necesidad de armonización contable.-}

El deseo de uniformidad contable, latente a lo largo del tiempo, se ha hecho apremiante en los últimos años. Las causas de estas urgencias en lograr una uniformidad de la información contable son múltiples. Merece destacarse la globalización de los negocios y de los mercados de capitales, así como, la creciente necesidad de financiación internacional que van adquiriendo las empresas. Las diferencias en los criterios y prácticas contables en los países se sustentan en los factores de orden económico, político, legal y social, los que tendrán que ser conciliados para poder implementar la armonización contable.

El uso de un sistema contable uniforme es importante para una competencia efectiva en los mercados de capitales, porque es improbable que los mercados sean capaces de evaluar, sobre la misma base, entidades que preparan su información económica y sus cuentas anuales de acuerdo con diferentes principios contables. Esta circunstancia ha motivado que los mercados de capitales exijan que los estados financieros de las entidades que operan en ellos, se preparen de acuerdo con los Principios de Contabilidad Aceptados en los países donde radican dichos mercados. $\mathrm{O}$ en todo caso, que los estados financieros de las entidades, preparados de acuerdo con los Principios de Contabilidad de sus respectivos países de origen, se acompañen de una conciliación de los resultados contables que presentan y los que se hubieran obtenido por aplicación de los Principios Contables del país en cuyo mercado quieren operar. Conciliación que, con frecuencia, arroja diferencias dramáticamente significativas entre los resultados.

La medición del patrimonio de una entidad debe lograrse con la máxima precisión posible, sin embargo, ante determinadas situaciones, diferentes profesionales pueden llegar a posiciones distintas. Existen varias razones para que esto ocurra, de las cuales mencionaremos sólo tres. La primera razón es que las condiciones de la economía no son las mismas en todo el mundo; la segunda, es que los objetivos de la información financiera no son los mismos para todos los usuarios; y la tercera, es que aún dentro de un mismo país, ciertos fenómenos económicos pueden ser vistos e interpretados de diferentes maneras. A la luz de estas divergencias, surge la siguiente pregunta: ¿Se necesitan normas nacionales e internacionales para un adecuado ejercicio de la profesión? La respuesta es afirmativa, dado que en el marco de la globalización existe un interés tanto local como internacional sobre la información financiera de las entidades. Quien espera ser entendido, debe expresarse en un lenguaje común, éstas son las normas contables. Se debe quizás conocer el por qué de las diferencias en las normas para comprender la magnitud del problema y proceder a la búsqueda de soluciones. Existen dos razones fundamentales que explican el por qué de las diferencias en las normas contables alrededor del mundo. La primera tiene que ver con la subjetividad inherente a la información financiera misma, éstos son complejos problemas inherentes a la profesión, para los cuales no necesariamente hay una sola respuesta correcta. La 
segunda tiene un efecto aún mayor y se relaciona con los ambientes económicos y legales de los distintos países; las diferencias en el entorno socieconómico entre cada país es también, uno de los obstáculos para la armonización de normas contables, y asimismo, explica el por qué una norma "importada" de un país puede fracasar en otro.

La armonización contable lograría pues, de forma inmediata, dos objetivos importantes. De una parte, evitaría que las sociedades tuvieran que preparar su información financiera, de acuerdo con los Principios de Contabilidad de su propio país y, adicionalmente, de acuerdo con los Principios de Contabilidad del país o países de los mercados en los que pretende operar $\mathrm{o}$, al menos, que tuvieran que elaborar una conciliación entre los resultados obtenidos con unos y otros principios. En segundo lugar, se evitaría que por un mismo ejercicio económico se puedan presentar resultados diametralmente opuestos dependiendo de qué Principios Contables se hayan aplicado, circunstancia esta última que dice muy poco a favor de la contabilidad.

Considero imprescindible que lleguemos a una unificación de los Principios de Contabilidad Generalmente Aceptados a nivel mundial. De esta forma, sólo se derivarían innumerables ventajas: las empresas que quisieran acudir a los mercados internacionales no tendrían que preparar más que un único juego de estados financieros, los inversores sólo tendrían que familiarizarse con unas normas de contabilidad, las auditorías serian más efectivas y los reguladores podrían estar seguros de que la información financiera que facilitacen las empresas sería de alta calidad y permitiría la comparación.

- Problemas frente a la armonización contable .- José Lainez Gadea plantea los siguientes problemas:
- Enfoque descriptivo-normativo es para elaborar la norma: el primero se da en base a la elección de alternativas contables con un grado de utilización y el segundo a la armonización de objetivos y escritura conceptual que se traducen en reglas generales.

- Cómo implementarla a nivel internacional, a través de organizaciones gubernamentales o profesionales.

- La rigidez o flexibilidad en la aplicación de las normas: dependiendo del entorno, la flexibilidad será mejor para su adecuación.

- La armonización de normas y entorno es más factible su aplicación en áreas geográficas con el mismo entorno, caso la Unión Europea.

- Flexibilidad o comparabilidad: son opuestos pero debe encontrarse el equilibrio, que acompañado por un enfoque normativo, bajo una estructura conceptual, permita la comparabilidad.

- Ámbito de aplicación de la norma: existe la disyuntiva de que si se aplica a las empresas multinacionales o a todas las empresas, recae la normalización y armonización en las primeras, y en segunda instancia, en las empresas nacionales.

- Instituciones contables.- La armonización contable surge por la apertura de las empresas al mercado internacional y como consecuencia de la necesidad de que se pueda establecer comparabilidad entre la información que ellas ofrecen. Han sido varios los organismos públicos y privados que se han preocupado por conseguir la armonización contable a nivel internacional $y$ a nivel regional. 


\section{De Atcance Internacional}

Organismos Públicos.- El Consejo Económico y Social de la Naciones Unidas (ONU) formó en julio de 1972 una comisión de expertos que se dedicaría al estudio de las empresas multinacionales y su influencia en el desarrollo económico mundial. En 1974 se creó la comisión de empresas transnacionales.

La OCDE (Organización de Cooperación y Desarrollo Económico) inició la normalización en el ámbito de la contabilidad nacional ampliándolos, posteriormente, al área de la microcontabilidad. En 1975 creó un comité de inversión internacional y de empresas multinacionales.

Organismos Privados.- Todo intento de armonización de las normas contables está amparado en congresos internacionales de profesionales. Desde 1957 se viene hablando de la necesidad de uniformidad en la presentación de los estados financieros emitidos por los diferentes países.

Se establece el ICCAP (Comité Internacional de Coordinación Contable). $\mathrm{Al}$ crearse otros organismos internacionales como el IFAC, el ICCAP cesó en sus funciones a partir de 1977.

El IASC (Comité Internacional de Normas Contable) fue creado como un comité dependiente del ICCAP. Se independizó al emitir su carta de constitución con nueve países como miembros fundadores, creándose el 29 de Junio de 1973. Los países que la formaron fueron: Austria, Canadá, Francia, Japón, México, Holanda, Reino Unido, Estados Unidos y República Federal de Alemania.

Federación Internacional de Contadores IFAC (Internacional Federation of Accountants) se constituyó en 1977 , por la desaparición del ICCAP y con el objetivo de armonizar la profesión. La IFAC tiene estrecha vinculación con el IASC, su objetivo básico es el desarrollo y perfeccionamiento de la profesión contable a nivel internacional alcanzando normas homogéneas.

Compromisos entre la IFAC y el IASC. La IFAC reconoce al IASC como el único cuerpo con responsabilidad y autoridad para emitir, a su nombre, pronunciamientos sobre normas internacionales de contabilidad, con plena autoridad de negociar y asociarse con otras organizaciones no miembros y para promover la aceptación y observancia mundial de tales normas.

\section{Đe Ateance Regional}

Organismos Públicos.- Uno de los mejores intentos de armonización, por parte de organismos cuyas normas tienen un ámbito regional, es la efectuada por la CEE (Comunidad Económica Europea), hoy UE (Unión Europea).

Organismos Privados. - Los organismos de carácter privado tuvieron que hacerse cargo de la armonización porque existía la necesidad de instrumentar mecanismos, que permitan reducir las discrepancias latentes entre los criterios y prácticas contables de los países situados dentro de un área geográfica. Estos organismos normalmente están formados por asociaciones profesionales.

La FEE (Federación de Expertos Contables Europeos), creada en 1986 por la fusión del Grupo de Estudios de Expertos Contables de la CEE (GEEC) y la UEC (Unión Europea de Expertos Contables, Económicos y Financieros) representan a la profesión contable europea a nivel internacional.

La IAA (Asociación Interamericana de Contabilidad), constituida en 1949, tiene su 
origen en la 1era. Conferencia Interamericana de Contabilidad, celebrada en San Juan de Puerto Rico. Su composición está dada por países de América, el Interamerican Accounting Asociation, hoy Asociación Interamericana de Contabilidad, AIC.

El CAC (Consejo Africano de Contabilidad), creado en 1979 por 23 países miembros de la OUA (Organización para la Unidad Africana); ha formado diversos comités y organismos de normalización y armonización contable en dichos países.

La CAPA (Confederación de Contables de Asia y el Pacífico) fue fundada en 1976 con ocasión de la celebración de la VIII Conferencia Asiática de Contabilidad.

ASEAN (La Federación de Contadores de la Asociación de Naciones del Sudeste Asiático), fue constituida en 1976 por 5 países (Indonesia, Malasia, Filipinas, Singapury Tailandia).

Otras Instituciones que emiten pronunciamiento y ejercen influencia en los países de América son:

AICPA (American Institute of Certified Public Accountants) que desde 1939 emiten los Accounting Principales Board: PCGA. Luego sería Financial Accounting Standars Board quien emite los pronunciamientos contables a través de los FASB. Junta de Normas de Contabilidad Financiera.

Instituto Mexicano de Contadores.

\section{- Situación actual de la armonización} contable.- Es necesario conocer el presente para planificar el futuro:

a) Vivimos en período de creciente apertura de mercados, de intensificación del comercio internacional e intercambio entre los países, movilidad de los capitales, que ha concluido en el fenómeno denominado "la globalización de la economía".

b) Esta globalización de la economía se ha traducido en inversiones desde y hacia los distintos países, creación de empresas multinacionales, etc. Lo que a su vez, ha significado nuevas necesidades de información que permitan evaluar la potencialidad de una inversión -mirando hacia el futuro- y la situación patrimonial, mirando el presente, pero en ambos casos desde un punto de vista diferente puesto que las inversiones se han hecho o se van a hacer en otros países.

c) La creación de mercados comunes entre los distintos países: Unión Europea, Nafta, Mercosur, han demostrado con mayor intensidad las necesidades de información antes indicadas y ha puesto en evidencia, la disparidad de criterios contables en lo relativo a formas de valuación, reconocimiento de los hechos económicos, medición de las variaciones patrimoniales, etc. que han derivado en estados financieros preparados bajo normativas contables diferentes que impiden su comparación y consolidación, obli. gando a su "reexpresión" para adecuarlos a otras normativas.

d) Esta disparidad de criterios contables ha demostrado que aún cuando existe una normativa contable internacional y un marco conceptual, que debiera servir de base a esa normativa, no ha existido en algunos países la preocupación de adecuar su normativa a la normativa internacional $o$, lo que es más grave, existe un desconocimiento de esa normativa.

e) Los países que integran los mercados comunes han hecho esfuerzos en solucionar estos problemas y han creado comisiones o subcomisiones, que se dedican al estudio de las normativas contables vigentes y a la 
armonización contable entre los países que forman el mercado regional.

f) En medio de este avanzar de la economía, en pleno periodo de su globalización, la contabilidad en América sigue basándose en "Principios de Contabilidad Generalmente Aceptados" y en Europa, aún cuando se dice que el balance debe presentar "a true and fair view" (una imagen fiel) de la empresa y su patrimonio, se utilizan normas similares a nuestros "Principios de Contabilidad", algunos algunos de los cuales se apartan de esa "imagen fiel" a la que se desea llegar.

No es tarea fácil lograr una armonización total por las naturales diferencias económicas, sociales y culturales de los países usuarios de la información contable; pero estamos seguros de que los profesionales contadores tienen la capacidad para enfrentar con éxito el desafío de lograr un lenguaje común que les permita un mutuo entendimiento y comunicación hacia el mundo donde deben actuar.

El proceso de armonización de normas contables a nivel internacional debe transitar por diversas etapas.En la mayoría de los casos, la premura de atención que exigen las aperturas vecinales hacen que las primeras actividades de integración se desarrollen a nivel regional. Analicemos por ejemploel caso del Mercosur, los pasos iniciales se fueron dando con los trabajos a nivel de comisiones dentro del denominado Grupode Integración del Mercosur conformado por los países miembros. Los primeros resultados sobre comparación de situaciones han evidenciado una gran variedad de asimetrías, destacándose como una de las más interesantes la referida al mismo concepto de norma.

El concepto de norma tiene diferentes interpretaciones en distintos países. Algunos adoptan la definición de norma, como una expresión de derecho positivo (Brasil y
Argentina). En cambio en Paraguay y Uruguay, el concepto de norma se equipara más al de recomendaciones para la práctica profesional. Esta diferente conceptualización de norma es una de las dificultades de mayor relevancia que enfrenta la profesión a nivel regional (p.e.: en Mercosur) y mundial, para la armonización contable.

Si queremos armonizar las normas contables, (y en concordancia con el distinguido Dr. Olivio Koliver), debemos antes que nada definir qué es una norma. Más adecuada es la de regla impositiva, de lo que implica la creación de:

- Un sistema de normas adecuadas al entorno económico que regirá.

- Un órgano que fiscalice su cumplimiento y establezca sanciones para aquellos que trans gredan lo que en ellas está establecido. Con organismos supranacionales reguladores y juzgadores de la actividad profesional dentro de la región habrá mayor confianza y seguridad.

El tema de armonizar las normas contables está presente en todo evento internacional desde el inicio de la presente déca$\mathrm{da}$, donde se pregona que es preciso que los contadores hablemos un mismo idioma para que los usuarios de la información financiera, sin importar su país de procedencia, puedan interpretarnos y comprendernos.

En los procesos evolutivos, deseados o no, crecen o sobreviven aquellos entes que son capaces de adaptarse; por lo tanto, en lo que a nuestra profesión se refiere, es exigible un proceso de adaptación a los cambios de la economía tanto a nivel gubernamental, como gremial e individual. Producto de estos tratados, la profesión contable reaccionó y comenzaron los estudios e implementaciones tendientes a la armonización del ejercicio profesional y sus normas. 
Ejemplo de ello lo constituye la creación de GIMECA, Grupo de Integración del Mercosur.

El costo de producir información, cumpliendo con las normas locales y las del país al que pertenece la casa matriz con quien consolida dicha información, es muy alto. El Grupo Intergubernamental de Expertos en Normas de Contabilidad y Presentación de Estados Financieros (ISAR) de las Naciones Unidas presentan informes anuales en relación con nuestra profesión y ejerce una función actual de armonización de normas a nivel mundial. Este grupo fue creado en 1982 por el Consejo Económico y Social, como una estructura organizacional permanente y actúa como regulador del ámbito internacional de consultas multilaterales sobre los problemas de contabilidad y presentación de informes de empresas transnacionales a nivel mundial. Asimismo, persigue la armonización de las normas a nivel internacional.

A nivel de las instituciones y organismos internacionales, la preocupación por la armonización de los aspectos de contabilidad son de primordial importancia ante los cambios y estrategias que se imponen en la vida económica de todos los países. Para estar a tono con estos cambios, es necesario una preparación firme y eficaz en los aspectos técnicos. Ningún país puede presumir de tener un único juego correcto de normas de contabilidad. Incluso en los Estados Unidos que tiene la historia más larga de emisión de normas de contabilidad y la mayor organización emisora de normas, que se caracteriza por elevados estándares de profesionalidad, sus normas muestran compromisos entre diferentes partes interesadas que pudieran haberse resuelto razonablemente de otra forma.

La UE no puede adoptar pasivamente cualesquiera normas y regulaciones consensuadas internacionalmente. Debemos contri- buir a dar forma a tales normas y regulaciones, participando con un sano criticismo.

Son muy positivos los esfuerzos que vienen realizando el IASC, la Organización Internacional de Comisiones de Valores, International Organization of Securities Commission, IOSCO y la UE en el campo de la armonización contable. Ahora todos los profesionales de la contabilidad debemos presionar para que los organismos aúnen esfuerzos, limen discrepancias y consigan la aplicación generalizada de las NIC's, de forma que puedan hacerse armonizables y comparables los estados financieros tanto de las empresas pertenecientes a los países que integran la Unión Europea, como las de aquellas que deseen acudir a los mercados internacionales.

Todos nuestros países están demostrando que tienen que funcionar en esa vía, y existen palpables esfuerzos en tal sentido, sin embargo, el trabajo hasta ahora es bastante lento en comparación con la velocidad de los cambios; es como si la globalización avanzara en carro y la armonización en bicicleta. La armonización debe efectuarse en forma científica y sin pérdida de tiempo.

\section{Papel de los colegios profesionales y de las universidades.}

Los colegios profesionales son los organismos responsables de la eficiencia en el desarrollo de la contabilidad y la entrega de información que la entidad en circunstancias económicas, jurídicas y culturales, requiere para el desarrollo de sus actividades. Tienen la responsabilidad de definir las normas por las cuales deben regirse los profesionales contadores de cada país. Los consejos profesionales que establecen o deberían establecer, las normas contables son los llamados a tomar las riendas de la armonización y a promulgar normas que sean compatibles con las de los demás países. 
Las universidades tienen mucho que aportar en el proceso de armonización contable, deben entregar el fruto de su investigación y su experiencia.

-IASC (Comité de normas internacionales de contabilidad).- El Comité de Normas Internacionales de Contabilidad -International Accounting Standards Committee- es un cuerpo privado independiente, con el objetivo de lograr uniformidad en los principios de contabilidad que son utilizados por los negocios y otras organizaciones en la información financiera alrededor del mundo. Desde 1983, los miembros de IASC han incluido a todos los cuerpos de contadores profesionales que son miembros de la IFAC.

\section{Los objetivos del IASC son:}

a) "Formular y publicar para el interés público, normas de contabilidad que deben de observarse en la presentación de estados financieros y el promover su aceptación mundial y su aplicación, y

b) Trabajar generalmente por el mejoramiento y armonización de reglamentaciones, normas de contabilidad y procedimientos relacionados a la presentación de estados financieros".

La IOSCO espera que el IASC proporcione NIC's (Normas Internacionales de Contabilidad) mutuamente aceptables, ya sean para las ofertas multinacionales de valores y otras ofertas internacionales. Los estados financieros se preparan y presentan para ser utilizados por usuarios externos de muchas empresas en todo el mundo. A pesar de que dichos estados financieros pueden parecer similares de un país a otro, existen diferencias que probablemente han sido causadas por una gran variedad de circunstancias de índole social, económica y legal. Tales circuns- tancias de diversa naturaleza han provocado la utilización de una variedad de definiciones de los elementos de los estados financieros.

El IASC se encuentra encargado de eliminar en la medida de lo posible tales diferencias, mediante la búsqueda de la armonización de regulaciones, principios contables y procedimientos relativos a la preparación y presentación de estados financieros. No obstante, su dificultad para la adopción total de las NIC's por los diferentes países, el IASC ha contribuido con éstas a la principal forma de armonización contable internacional, utilizándolas sobre todo en el acercamiento de los inversionistas a la información contable internacional que permita la globalización de la economía. De ahí que muchos argumenten que sería mejor referirse al IASC como el organismo más adecuado para el establecimiento de normas de contabilidad, y utilizar sus normas como una base de armonización dentro de la UE.

Para ello, es necesario que el IASC siga trabajando en la supresión de opciones alternativas dentro de sus normas, labor en la que ha conseguido un gran avance, según revisión llevada a cabo en los últimos años.

- NIC"s (Normas Internacionales de Contabilidad).- Los Principios de Contabilidad Generalmente Aceptados, hoy en día son aplicados como normas internacionales de contabilidad. Es decir, de normas de naturaleza general, se pasa al estudio de problemas de registro y tratamiento de partidas específicas, en los cuales las NIC's sin ser deterministas, otorgan al encargado de elaborar la información, las herramientas suficientes como para poder, usando su criterio profesional, enfrentar cualquier clase de transacción que realice la entidad contable, a fin de poder interpretarla correctamente y poder reflejarla en los reportes contables que le sean requeridos. 
Asimismo, paralelamente a los procedimientos recomendados a seguir en el tratamiento de las operaciones, se establecen criterios alternativos, entre los cuales el profesional contable tendrá que elegir de acuerdo a las necesidades de información que requiere atender. Lógicamente, al expandirse los negocios a nivel mundial, se han generado hechos económicos que tienen que ser registrados contablemente. Por ello, hoy surge la primera necesidad la de hablar el mismo idioma contable, es decir, que lo que se contabiliza en el Perú, corresponda a los mismos criterios de la contabilización de cualquier otro país, por esto es necesario aplicar NIC's emitidas por el IASC.

Para tener éxito en esta época globalizante, es preferible tener una mentalidad global, entender que nuestros problemas no son sólo del país, sino del mundo. Tener una mentalidad global es tener como nivel de vida el mejoramiento continuo, y como dijo Gary Hamel: "El futuro no se puede predecir, hay que crearlo".

Oficialización de las Normas Internacionales de Contabilidad en el Perú. Mediante Resoluciones $\mathrm{CNC} \mathrm{N}^{\circ}$ 005-94-EF/93.01 (30.03.1994), $\mathrm{N}^{\circ} 007-96-\mathrm{EF} / 93.01(31.07$. 1996), No 011-97-EF/93.01 (01.12.1997) y $\mathrm{N}^{\circ} 014-98-\mathrm{EF} / 93.01(06.11 .1998) \mathrm{el}$ Consejo Normativo de Contabilidad, considerando la necesidad de la internacionalización de los principios y técnicas contables que incluyan criterios comunes que favorezcan la comparabilidad, oficializó los acuerdos adoptados en los Congresos Nacionales de Contadores Públicos para la aplicación de las NIC's. Asimismo, precisa que los estados financieros deben prepararse observando su aplicación y recomendar su divulgación a nivel nacional por los colegios profesionales, facultades de ciencias contables y entidades representativas de la profesión, en coordinación con la Contaduría Pública de la Nación.
De otro lado, la Ley General de Sociedades -vigente a partir del $\mathrm{I}^{\circ}$ de enero de 1998- dispone que los estados financieros se preparen de conformidad con las disposiciones legales y los Principios de Contabilidad Generalmente Aceptados. Mediante Resolución $\mathrm{N}^{\circ}$.013-98-EF/93.01 del Consejo Normativo de Contabilidad, se precisa que los "Principios de Contabilidad Generalmente Aceptados" comprende por extensión substancialmente a las Normas Internacionales de Contabilidad y, en forma supletoria, se podrá emplear los principios de contabilidad aplicados en los Estados Unidos de Norteamérica.

Las NIC's oficializadas para su aplicación son:

01 al 13. Aprobada por Resolución $\mathrm{N}^{\circ} 039$ del X Congreso Nacional - 1986, Lima. 14 al 23. Aprobada por Resolución $\mathrm{N}^{\circ} 012$ del XI Congreso Nacional - 1988, Cuzco. 24 al 29. Aprobada con Resolución $N^{\circ} 01$ del XII Congreso Nacional - 1990, Cajamarca.

30 al 31 . Aprobada con Resolución $\mathrm{N}^{\circ} 28$ del XIII Congreso Nacional - 1992, Tacna. 32. Aprobada con Resolución $\mathrm{N}^{\circ} 15 \mathrm{del} \mathrm{XV}$ Congreso Nacional - 1996, Huánuco.

33 y 34 . Aprobada con Resolución $N^{\circ} 22$ del XVI Congreso Nacional - 1998, Tumbes.

A partir de 1999 entran en vigencia las NIC's 35 al 38.

A continuación se señala un inventario de las NIC's :

NIC 1. Presentación de estados financieros.

NIC 2. Existencias.

NIC 3. Estados financieros consolidados. (Reemplazada por la NIC 27 y 28).

NIC 4. Tratamiento contable de la depreciación.

NIC 5. Información que debe revelarse en los estados financieros. 
NIC 6. Tratamiento contable de los precios cambiantes (Reemplazada por la NIC 15).

NIC 7. Estado de flujos de efectivo.

NIC 8. Utilidad o pérdida neta del ejercicio. Errores sustanciales, y cambios en las políticas contables.

NIC 9. Costos de investigación y desarrollo.

NIC10. Contingencias y hechos ocurridos después de la fecha del balance.

NIC 11. Contratos de construcción.

NIC 12. Impuesto a la renta.

NIC 13. Presentación de activos y pasivos corrientes.

NIC 14. Presentación por segmentos.

NIC 15. Información que refleja los efectos de los precios cambiantes.

NIC 16. Inmuebles, maquinaria y equipo.

NIC 17. Arrendamientos.

NIC 18. Ingresos.

NIC 19. Costo de las prestaciones de jubilación.

NIC 20. Tratamiento contable de los subsidios gubernamentales y revelaciones referentes a la asistencia gubernamental.

NIC 21. Efecto de las variaciones en los tipos de cambio.

NIC 22. Fusión de negocios.

NIC 23. Costos de financiamiento.

NIC 24. Revelaciones sobre entes vinculados.

NIC 25. Tratamiento contable de las inversiones.

NIC 26. Tratamiento contable y presentación de información sobre planes de prestaciones de jubilación.

NIC 27. Estados financieros consolidados y tratamiento contable de las inversiones en subsidiarias.

NIC 28. Tratamiento contable de las inversiones en compañías asociadas.

NIC 29. Presentación de información financiera en ambientes de economía hiperinflacionaria.

NIC 30. Revelaciones en los estados financieros de bancos e instituciones financieras.

NIC 31. Información financiera sobre participaciones en asociaciones en participación.

NIC 32. Títulos financieros: Revelaciones y presentación.

NIC 33. Utilidades por acción.

NIC 34. Informes financieros intermedios.

NIC 35. Operaciones discontinuadas.

NIC 36. Deterioro de activos.

NIC 37. Provisiones, pasivos contingentes y activos contingentes.

NIC 38. Activos intangibles.

\section{CONCLUSIONES}

1.- Ante la globalización, no se trata de estar a favor o en contra, sino de prepararse para operar eficientemente bajo esa nueva circunstancia. Si ignoramos la lluvia, nos mojamos. Y si como profesionales, empresarios o nación pasamos por alto la globalización, nos quedaremos atrasados, empobrecidos y seremos desplazados.

2.- En una economía mundial que se caracteriza por la globalización de los negocios, el intercambio de información financiera emitida sobre bases homogéneas, se convierte en una necesidad imprescindible.

3.- La armonización contable será posible gracias a la participación de los organismos profesionales que logren a través de congresos, conferencias o convenciones, establecer la necesidad de utilizar un lenguaje universal en la contabilidad.

4.-La armonización contable internacional será posible en la medida que se concilien criterios, conceptos contables fundamentales y prácticas generales para alcanzar la uniformidad de la información, que permita su comparabilidad en la globalización económica actual. La armonización de modelos y prácticas contables a nivel internacional deben tener como principal objetivo la comparabilidad de los estados financieros. 
5.-Esta armonización debe ser llevada a cabo por un organismo internacional emisor de normas, pues no podemos olvidar que no existe una sola nación que tenga un juego de normas contables con los méritos suficientes para ser adoptadas por el mundo entero. En la actualidad, el único organismo emisor de normas de contabilidad con carácter internacional es el IASC, quien emite las NIC's.

6.- Respetando siempre el criterio uniformizado de las normas, se puede llegar a la formulación de estados financieros perfectamente entendibles y comparables a nivel internacional, con todas las consecuencias económicas, financieras, e inclusive políticas que ello implica.

7,- El paradigma de la utilidad de la información financiera ha ampliado el alcance de las normas contables. A las tradicionales normas de medición del beneficio, se suman ahora nuevas normas de exposición, incorporados a las mismas cuestiones, tales como el concepto de responsabilidad social y de protección del medio ambiente.

\section{BIBLIOGRAFIA}

FEDERACIÓN DE COLEGIOS DE CONTADORES PÚBLICOS DEL PERÚ Y SU JUNTA DE DECANOS.

Normas internacionales de contabilidad, 1997. Lima - Perú.

FORO INTERNACIONAL DE CONTABILIDAD. Asociación interamericana de contabilidad. Lima-Perú,1996.

HERRERA H., Juan

"El papel de la IFAC y el IASC en la armonización contable a nivel mundial". En: Revista Interamericana $N^{\circ}$ 57. 1997.
LAINEZ G., José.

Comparabilidad internacional de la información financiera, análisis y posición de la normatividad española. Instituto de Contabilidad y Auditoría de Cuentas. Madrid - España, 1993.

SANTISOH., L.

"Un modelo contable globalizador. El modelo de valores económicos-contables". En: Revista Brasilera de Contabilidade, Brasilia-Brasil, 1996.

TUA PEREDA, Jorge

Principios y normas de contabilidad. Instituto de Planificación Contable, Madrid España, 1983

XXII CONFERENCIA INTERAMERICANA DE CONTABILIDAD.

Asociación interamericana de contabilidad, Lima - Perú, 1997. 